\title{
Curcumin Reduces Cognitive Deficits by Inhibiting Neuroinflammation through the Endoplasmic Reticulum Stress Pathway in Apolipoprotein E4 Transgenic Mice
}

\author{
Jiejian Kou, Minghui Wang," Junzhuo Shi, Haiyu Zhang, Xiaohui Pu, Shiyong Song, Cuiling Yang, \\ Yi Yan, Yvonne Döring, Xinmei Xie,* and Xiaobin Pang*
}

Cite This: ACS Omega 2021, 6, 6654-6662

Read Online

ABSTRACT: Apolipoprotein E4 (ApoE4) is the main genetic risk factor for Alzheimer's disease $(\mathrm{AD})$, but the exact way in which it causes $\mathrm{AD}$ remains unclear. Curcumin is considered to have good therapeutic potential for $\mathrm{AD}$, but its mechanism has not been clarified. This study aims to observe the effect of curcumin on ApoE4 transgenic mice and explore its possible molecular mechanism. Eight-month-old ApoE4 transgenic mice were intraperitoneally injected with curcumin for 3 weeks, and the Morris water maze test was used to evaluate the cognitive ability of the mice. Immunofluorescence staining, immunohistochemistry, western blotting, and enzyme-linked immunosorbent assay (ELISA) were used to examine the brain tissues of the mice. Curcumin reduced the high expression of ApoE4 and the excessive release of inflammatory factors in ApoE4 mice. In particular, the expression of marker proteins of endoplasmic reticulum (ER)

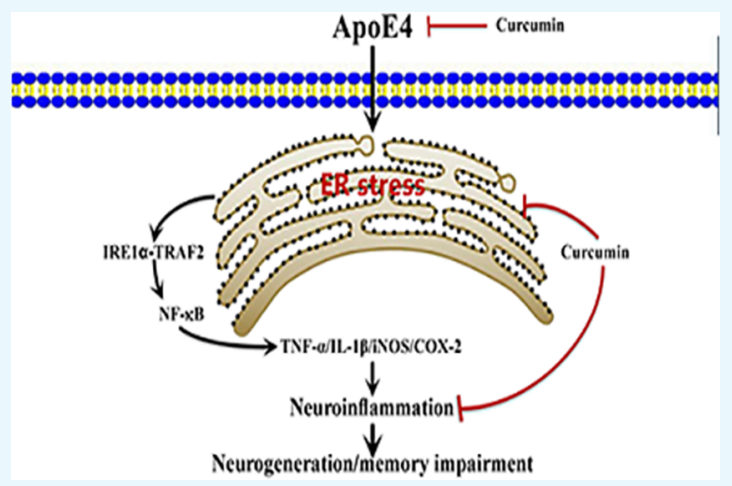
stress was significantly increased in ApoE4 mice, while curcumin significantly reduced the increase in the expression of these proteins. Collectively, curcumin alleviates neuroinflammation in the brains of ApoE4 mice by inhibiting ER stress, thus improving the learning and cognitive ability of transgenic mice.

\section{INTRODUCTION}

Alzheimer's disease $(\mathrm{AD})$ is a neurological disease characterized by profound memory loss and progressive dementia and is the most common cause of dementia in the elderly. More than 50 million patients worldwide are affected by $\mathrm{AD}$, and this number is expected to quadruple by 2050. Alzheimer's disease is a serious and unresolved economic and public health problem, and there is an urgent need to develop effective treatments to prevent its development. ${ }^{1,2}$

In addition to amyloid plaques (APs) formed by $\beta$-amyloid peptides (A $\beta$ ) and neurofibrillary tangles (NFTs) caused by Tau protein phosphorylation, ${ }^{3}$ neuroinflammation, and neuronal degeneration are the main features of $\mathrm{AD} .{ }^{4,5}$ Although the main causes of neuronal death in $\mathrm{AD}$ are generally believed to be APs and NFTs, it is clear that other biological response modulators, including local chronic inflammatory responses mediated by glial cells, also have an impact on the pathological process. $^{6}$

Apolipoprotein E (ApoE), a 299-amino-acid protein with a molecular weight of $34 \mathrm{kDa}$, plays an important role in lipid and cholesterol transport and lipoprotein metabolism in the central and peripheral nervous systems. ApoE has three alleles, $\varepsilon 2, \varepsilon 3$, and $\varepsilon 4$. Among them, the $\varepsilon 4$ allele (ApoE4) is the main genetic risk factor for $\mathrm{AD},{ }^{7}$ which has been recognized for decades; however, the role of ApoE4 in the pathogenesis of $\mathrm{AD}$ has not yet been elucidated. In recent years, ApoE4 has been found to be a major genetic risk factor for a variety of inflammatory diseases, including atherosclerosis, diabetes, and Alzheimer's disease. ${ }^{8-11}$

Sustained endoplasmic reticulum (ER) stress is considered a pathological driver of many chronic diseases because it may cause abnormal inflammatory signals and promote cell death. ${ }^{12}$ In neurodegenerative disease, the accumulation of misfolded proteins in neurons and associated ER stress can lead to neuronal dysfunction. ${ }^{13}$ In addition, ER stress may promote disease progression by enhancing the destructive inflammatory response. However, the specific mechanism of ER stressmediated neuroinflammation is still unknown. ${ }^{14}$

Curcumin (molecular structure is shown in Figure S1) is a polyphenolic substance extracted from turmeric, a plant of the curcuma family. ${ }^{15}$ Toxicity studies have shown that it is quite safe even at high doses (up to $12 \mathrm{~g}$ in humans). ${ }^{16}$ Curcumin's

Received: November 8, 2020

Accepted: February 8, 2021

Published: March 2, 2021 
A
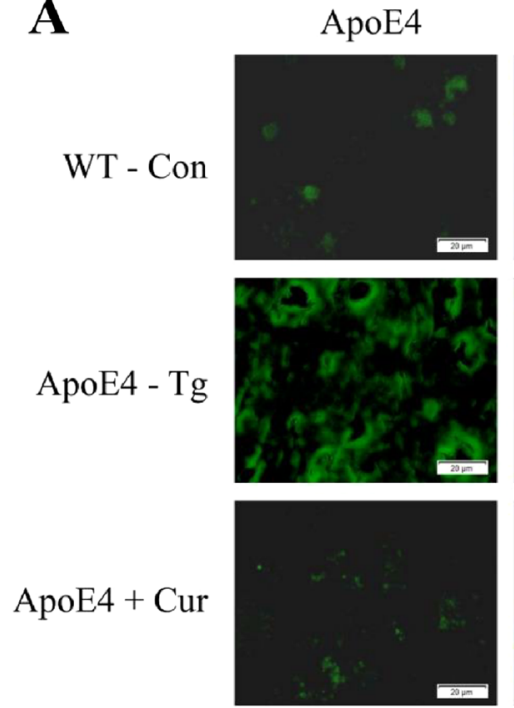
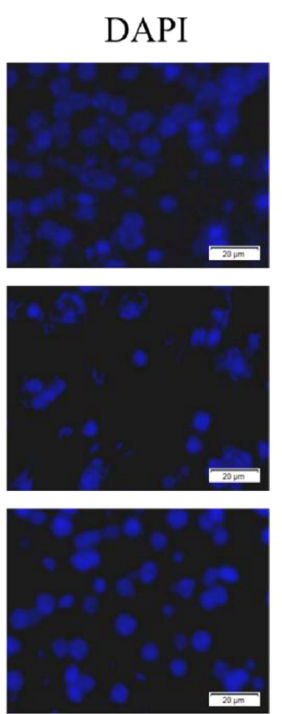
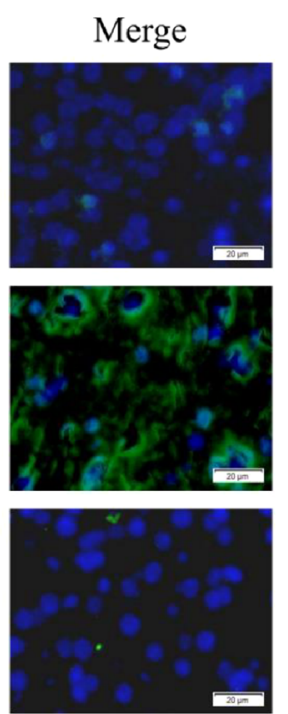

B

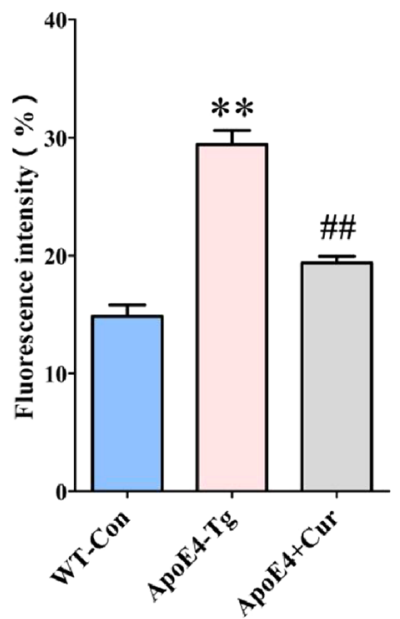

Figure 1. ApoE4 expression is enhanced in transgenic mice. (A) Localization of ApoE4 in the brains of each group of mice. Scale bar $=20 \mu \mathrm{m}$. (B) Quantification based on the average positive area. Data are expressed as the mean \pm standard error of the mean $(\mathrm{SEM}) n=3 .{ }^{*} p<0.05$ versus WTCon; $\# p<0.05$ versus ApoE4-Tg.
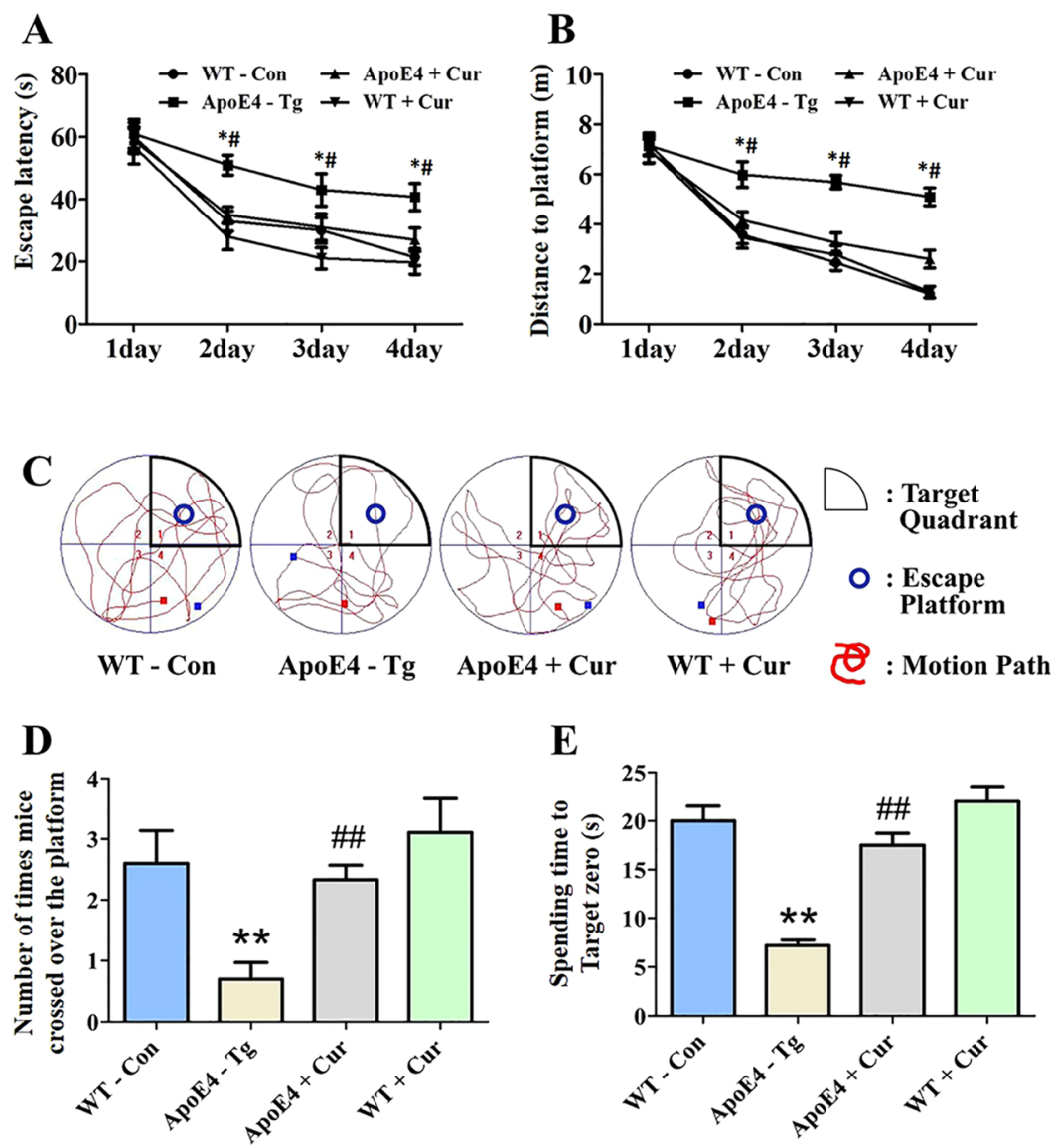

Figure 2. Curcumin ameliorated learning and memory impairment in ApoE4-Tg mice. The training trials were performed three times a day for 4 days. The swimming distance and swimming time before arrival at the platform were automatically recorded. Twenty-four hours after the training trials, a probe test was performed. (A) Time to reach the hidden platform. (B) Total swimming distance during the trial. (C) Tracks of the mice in the probe trial on the fifth day. (D) Number of times the mice crossed over the platform location in the probe trial. (E) Duration of time spent in the target zone area in the probe trial. All values are presented as the mean \pm SEM $n=6$. ${ }^{*} p<0.01$ versus WT-Con; \#p $<0.05$ versus ApoE4-Tg.

pharmacological action is exerted at multiple sites, including enzymes, transcription factors, growth factors, neurotransmitter receptors, inflammatory mediators, and a large number of protein kinases. It has anti-inflammatory, antitumor, antioxidative, and antibacterial effects. ${ }^{17}$ Curcumin has been used as a condiment in food in Southeast Asia for centuries. ${ }^{18}$ 

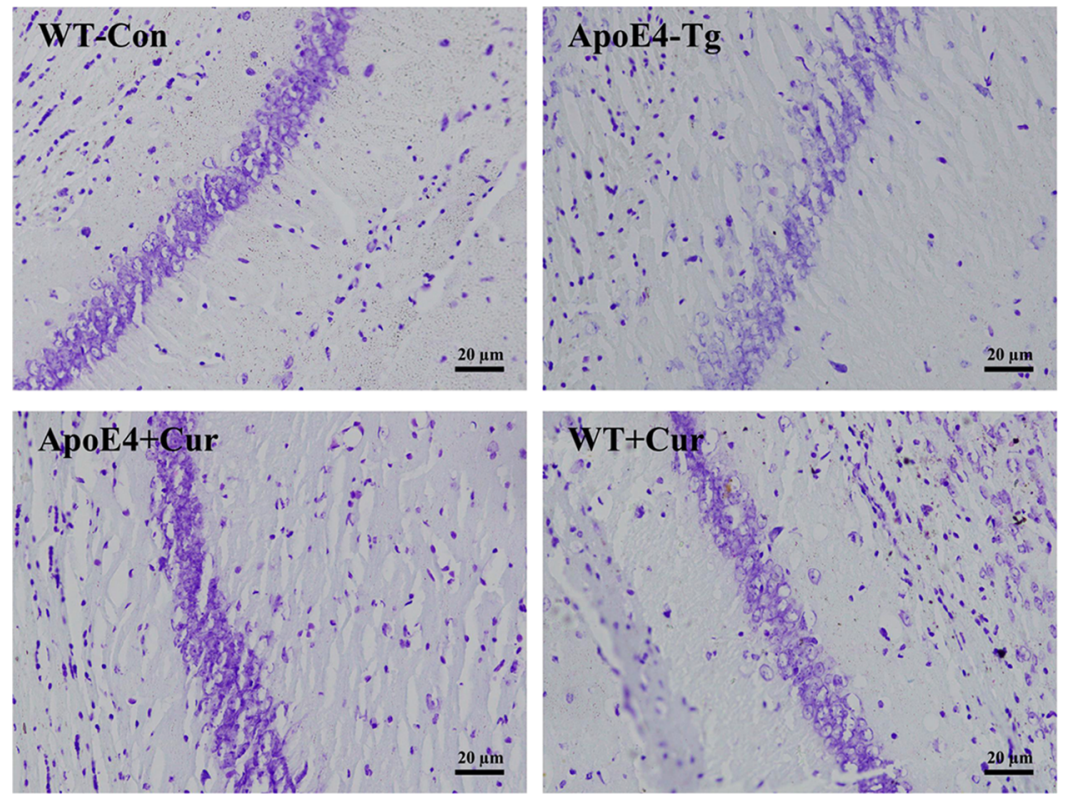

Figure 3. Curcumin alleviated the pathological changes in neuronal cells in ApoE4-Tg mice. Nissl staining of the neuronal cells. Representative images were chosen from each experimental group. Scale bar $=20 \mu \mathrm{m}$.

According to the initial epidemiological analysis of the Indian population, curcumin has a strong potential to treat $\mathrm{AD} .{ }^{19}$ The incidence of $\mathrm{AD}$ was 4.4-fold lower in long-term curcumin users than those without curcumin in the United States, but whether curcumin affected ER stress in $\mathrm{AD}$ remains unclear. ${ }^{20}$

In this study, ApoE4 transgenic mice were used as model animals of $\mathrm{AD}$ to observe the effect of curcumin and to investigate whether curcumin can inhibit neuroinflammation in ApoE4 transgenic mice by inhibiting ER stress, thereby improving the cognitive ability of the model mice.

\section{RESULTS}

2.1. Curcumin Reduced the High Expression of ApoE4 in Transgenic Mice. Immunofluorescence staining showed that the expression of ApoE4 was enhanced in the brains of transgenic mice, while curcumin reduced the high expression of ApoE4 in the brains of transgenic mice to a level similar to those of wild-type (WT) control mice (Figure 1).

2.2. Curcumin Reduced Spatial Learning Deficits in ApoE4-Tg Mice. The Morris water maze test is a widely accepted method to examine cognitive function, and it was used to evaluate the spatial learning and memory abilities of mice in this study. All mice were trained for 4 days and tested three times a day. In the training phase, each group showed an effect on learning. Compared with those in the WT-Con group and the WT + Cur group, mice in the ApoE4-Tg group required a longer time and distance to reach the platform, while those in the ApoE4 + Cur group showed a shorter incubation period and escape distance, which was significantly different from those of the transgenic mice group (Figure 2A,B).

After the water maze test, we conducted a probe test to investigate memory retention. In the probe test, the time spent in the platform area and the number of times that the mice crossed the platform were all significantly reduced in ApoE4$\mathrm{Tg}$ mice compared to those in the WT-control group, which could be restored in ApoE4-Tg mice after curcumin treatment (ApoE4 + Cur) (Figure 2C-E).
2.3. Curcumin Reduced Neuronal Cell Death in ApoE4-Tg Mice. The survival rate of neurons was determined by crystal violet staining. In the WT-control group and the WT + cur group, neurons in the hippocampal and cortical regions exerted good morphology and were closely arranged; in contrast, neurons in the ApoE4-Tg group were not well defined. Moreover, compared with those in the WT-control group, the number of neurons in the ApoE4-Tg group was significantly reduced, while the cell morphology and the number of neurons in the ApoE4 + cur group were improved and significantly increased, respectively (Figure 3 ).

2.4. Curcumin Alleviated Cerebral Inflammation in ApoE4-Tg Mice. To determine the neuroinflammatory response in ApoE4 mice, we detected the expression of COX-2 and iNOS by immunofluorescence staining and the levels of TNF- $\alpha$ and IL- $1 \beta$ by ELISA. The immunofluorescence results showed that the expressions of COX-2 and iNOS in the brains of ApoE4-Tg mice were significantly higher than those in the brains of WT mice, and curcumin treatment significantly inhibited COX-2 and iNOS expressions in ApoE4Tg mice (Figure 4A,B). Our results also showed that the levels of TNF- $\alpha$ and IL- $1 \beta$ in the brains of ApoE-Tg mice were significantly higher than those in the brains of WT mice, which could be reduced by the treatment of curcumin in ApoE4-Tg mice (Figure 4C,D).

2.5. Curcumin Inhibited the Activation of NF- $\kappa B$ in ApoE4-Tg Mice. NF- $\kappa \mathrm{B}$ is a critical transcription factor that regulates the inflammatory response, so we examined the activation of NF- $\kappa \mathrm{B}$ in ApoE4-Tg mice. The western blotting results showed that the translocation of the NF- $\kappa \mathrm{B}$ subunit $\mathrm{p} 65$ to the nucleus was obviously enhanced in ApoE4-Tg mice compared with that in WT mice, while curcumin inhibited the nuclear translocation of p65 in ApoE4 mice (Figure 5).

2.6. Curcumin Inhibited Endoplasmic Reticulum (ER) Stress in ApoE4-Tg Mice. We detected the expression of the marker proteins of ER stress in mice by immunohistochemistry. The results showed that positive staining of GRP78 or IRE1 $\alpha$ cells in ApoE4-Tg mice was increased significantly, 
A

WT - Con ApoE4 - Tg ApoE4 + Cur WT + Cur
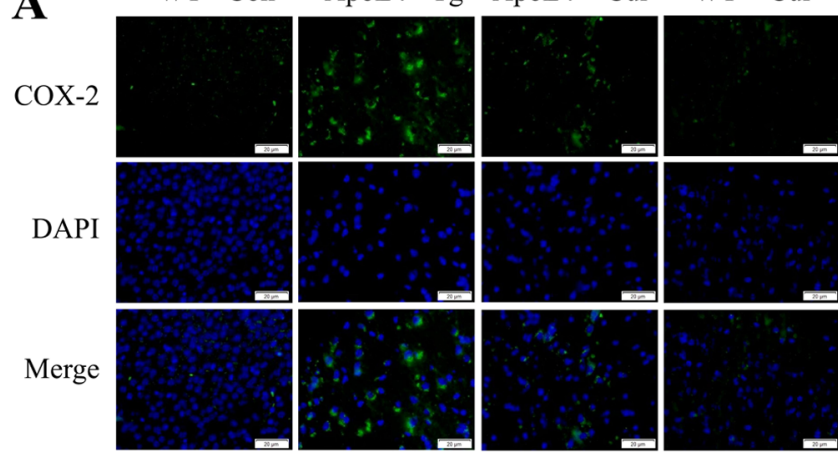

B

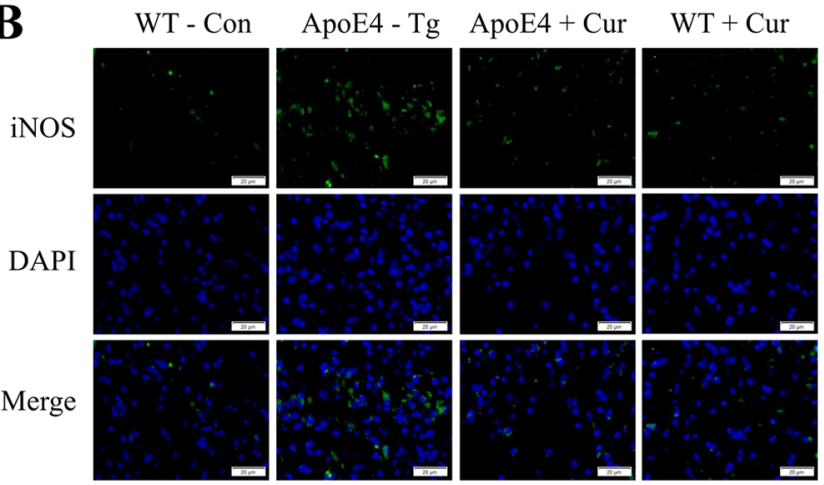

C
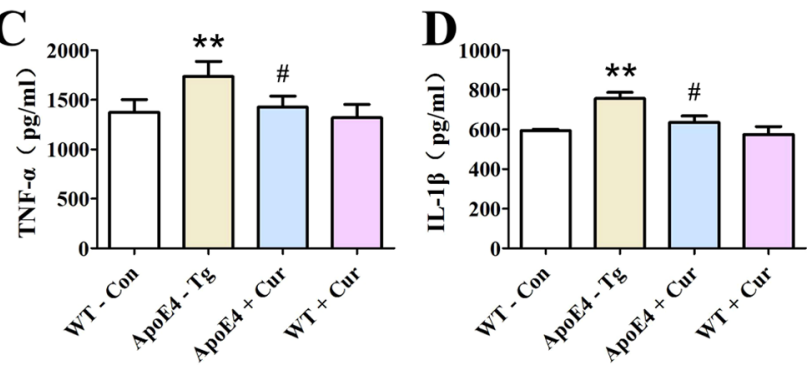

Figure 4. Effects of curcumin on the release of proinflammatory cytokines in ApoE4-Tg mice. Expressions of COX-2 (A) and iNOS (B) in mice are determined by immunofluorescence staining. Representative images of stained tissues are captured using a microscope. Scale bar $=20 \mu \mathrm{m}$. Levels of TNF- $\alpha$ (C) and IL- $1 \beta$ (D) are detected by ELISA. Data are expressed as the mean $\pm \operatorname{SEM} n$ $=3$. $* *<0.01$ versus WT-Con; \# $p<0.05$ versus ApoE4-Tg.

suggesting that ER stress occurred in ApoE4-Tg mice. However, the curcumin treatment significantly reduced the number of GRP78-positive and IRE1 $\alpha$-positive cells in transgenic mice (Figure 6A).

Next, the expressions of GRP78 and IRE1 $\alpha$ proteins were determined by western blotting. Consistent with the immunohistochemical results, their expressions in ApoE4-Tg mice were significantly higher than those in wild-type mice, while curcumin treatment inhibited the increase in the expression of these two proteins (Figure 6B).

\section{DISCUSSION}

ApoE4 is the most important genetic risk factor for sporadic $\mathrm{AD} .{ }^{21}$ Individuals with one or two ApoE4 alleles have a 4-8fold increased risk of $\mathrm{AD}$, and the onset age of $\mathrm{AD}$ is $7-15$ years earlier for these individuals. Studies have shown that the brain physiology of ApoE4 carriers changes years before clinical markers, such as $\mathrm{A} \beta$ and tau pathology and memory deficits, appear. ${ }^{22}$ Similarly, mice expressing human ApoE4 showed signs of impaired learning and memory, neurodegeneration, ${ }^{23}$ and increased amyloid- $\beta$ ( $\left.\mathrm{A} \beta\right)$ peptide accumulation and subsequent deposition in the brain. ${ }^{24}$ Besides, studies of Lewandowski et al. ${ }^{25}$ and Shi et al. ${ }^{26}$ strongly suggested ApoE4 transgenic mice as model animals for Alzheimer's disease. In our study, compared with those of the wild-type mice, the learning and memory abilities of the ApoE4 transgenic (ApoE4-Tg) mice were significantly decreased, and the dramatic loss of neurons in the brain of this model confers the link between ApoE4 and AD.

Studies have shown that ApoE4 is a major genetic risk factor for atherosclerosis, vascular dementia, ${ }^{27}$ diabetes, ${ }^{28}$ and other diseases that are closely related to inflammation. Therefore, the relationship between ApoE4 and inflammation has drawn increasing attention. Pathological changes caused by neuroinflammation occur in the brains of patients with $\mathrm{AD},{ }^{29}$ and it has been recognized that abnormal inflammatory processes in the central nervous system (CNS) can lead to neurological dysfunction; ${ }^{30}$ neuroinflammation plays an important role in the degeneration of $\mathrm{AD}$ neurons and is involved in the vicious cycle of $\mathrm{A} \beta$ deposition, neuron damage, tangle formation, and death. $^{31}$ The results of this study showed that the release of inflammatory cytokines in the brains of ApoE4 mice was significantly increased compared with that in the brains of wild-type mice, suggesting that ApoE4 is closely related to neuroinflammation in the central nervous system.

ER stress results in a protective cellular stress response by activating the unfolded protein response (UPR) and other signaling pathways in response to various damaging conditions. ${ }^{32,33}$ However, under the conditions of continuous ER

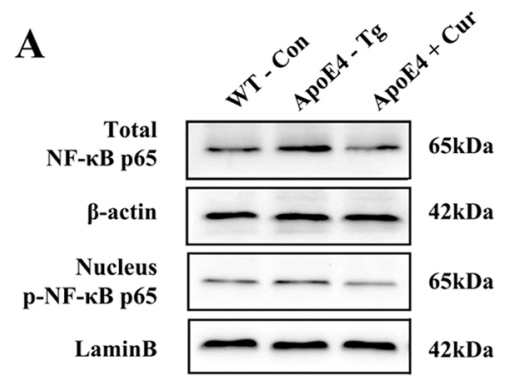

B

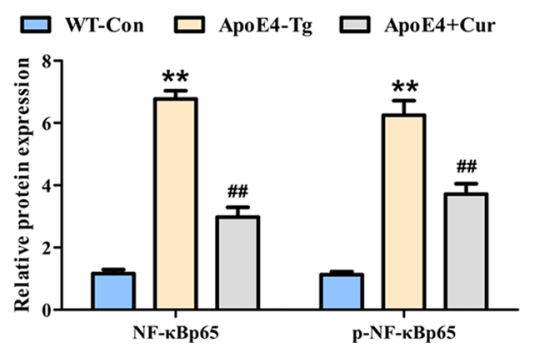

Figure 5. Effects of curcumin on NF- $\kappa$ B activity in ApoE4-Tg mice. (A) Expression of $p 65$ in the total and nuclear fractions is determined by western blotting analysis. (B) Protein expression is normalized to that of $\beta$-actin or lamin $\mathrm{B}$, and the relative densities were normalized to those of the WT mice. The values presented are the mean \pm SEM $n=3$. \#\#p $<0.01$ versus WT-Con; ** $p<0.01$ versus ApoE4-Tg. 

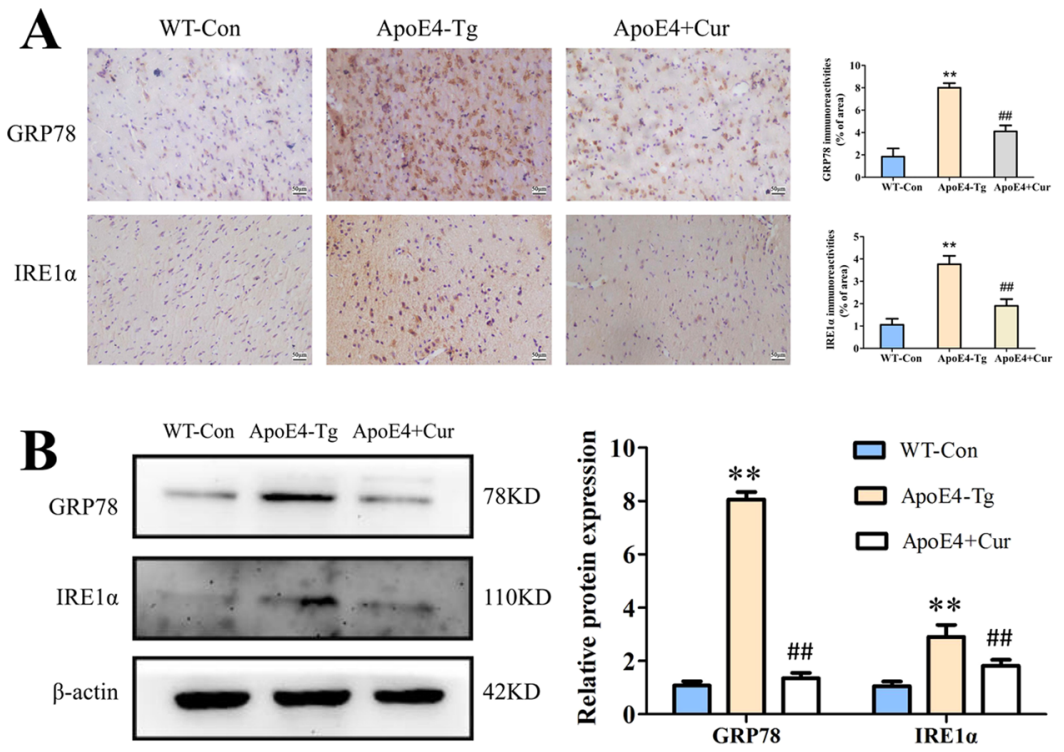

Figure 6. Expression of ER stress marker proteins in ApoE4-Tg mice. (A) Expressions of GRP78 and IRE1 $\alpha$ in WT and ApoE4-Tg mice were determined by immunohistochemistry. Representative images of stained tissues were captured using a microscope. Scale bar $=20 \mu \mathrm{m}$. $(\mathrm{B})$ Expressions of GRP78 and IRE1 $\alpha$ were detected through western blotting using specific antibodies in mouse brain tissues. The values presented are the means $\pm \operatorname{SEM} n=3$. \#\#p 0.01 versus WT-Con; $* * p<0.01$ versus ApoE4-Tg.

stress, cells will reduce self-protection and initiate cell death signaling. It has been reported that ER stress induces $\mathrm{A} \beta$ to contribute to neurodegeneration in $\mathrm{AD} .{ }^{34} \mathrm{In}$ addition, several reports have described the activation of the ER stress response in $\mathrm{AD}$ mouse models. Normally, glucose-regulated protein 78 (GRP78) binds to inositol-requiring enzyme $1 \alpha$ (IRE1 $\alpha){ }^{35}$ protein kinase-like endoplasmic reticulum kinase (PERK), and activated transcription factor 6 (ATF6) to form a complex and remains inactive. When ER stress is induced by injury, GRP78 dissociates from PERK, IRE1 $\alpha$, and ATF6 to initiate the $\mathrm{UPR}^{36}$ Therefore, GRP78 is regarded as an important marker for ER stress. In our study, expression of GRP78 was higher in ApoE4-Tg mice than in wild-type mice, confirming the activation of ER stress in ApoE4-Tg mice. In mammalian cells, the mediators involved in triggering the UPR response are IRE1 $\alpha$, PERK, and ATF6, ${ }^{37,38}$ each of which activates a different signaling pathway to produce the corresponding effect. ${ }^{39}$ During the ER stress response, after oligomerization, IRE $1 \alpha$ recruits the adapter protein tumor necrosis factor- $\alpha$ receptor-associated factor 2 (TRAF2), and the formation of the IRE $1 \alpha$-TRAF 2 complex mediates the activation of NF- $\kappa \mathrm{B}$ signaling pathways. ${ }^{40-45}$ NF- $\kappa \mathrm{B}$ is a transcription factor that initiates and regulates the expression of several inflammatory processes that are attributed to the pathology of several neurodegenerative diseases. When the NF- $\kappa \mathrm{B}$ signaling pathway was activated, it would cause the increase of inflammatory factors, such as IL-6, TNF- $\alpha$, etc. ${ }^{46}$ Therefore, there seems to be an interesting relationship between ER stress and neuroinflammation. In our study, compared with wild-type mice, the expressions of inflammatory cytokines in the brain of ApoE4-Tg mice were increased dramatically. In addition, our results also showed that the NF- $\kappa \mathrm{B}$ signaling pathway was activated in the brains of ApoE4-Tg mice, accompanied by a higher expression of IRE $1 \alpha$ compared to wild-type control mice. However, we did not investigate on the ATF6-mediated pathway in ER stress, and the expression of the IRE $1 \alpha$ pathway downstream player TRAF2 was also not examined. Hence, we were unable to decipher the effect of curcumin on the precise network in ER stress, which could be the limitations of our study.

Curcumin, as an effective anti-inflammatory small molecule, was shown to inhibit the COX-2 expression in human colon cancer cells. ${ }^{47}$ It also alleviated LPS-induced inflammation by switching a proinflammatory M1 microglial to an antiinflammatory M2 phenotype via reducing the imbalance of TREM2 and TLR4 and balancing the downstream NF- $\kappa \mathrm{B}$ activation. ${ }^{48}$ Recently, Wang et al. ${ }^{49}$ have proven that curcumin can inhibit ApoE4-induced injury by upregulating the expression of peroxisome proliferator-activated receptor- $\gamma$ to inhibit the activation of NF- $\kappa \mathrm{B}$ signaling in SH-SY5Y cells. Apart from the anti-inflammatory role, curcumin also acts as a known antioxidant that scavenges reactive oxygen species (ROS). Zheng et al. found that curcumin can protect SH-SY5Y cells against appoptosin-induced intrinsic caspase-dependent apoptosis by increasing heme oxygenase- 1 expression and reducing ROS production. ${ }^{50}$ Chhunchha et al. ${ }^{51}$ also proposed that curcumin could reinforce the naturally occurring Prdx6 expression, attenuate ROS-based ER stress and NF- $\kappa$ Bmediated aberrant signaling, then improve the survival of mouse hippocampal cells (HT22), and may provide an avenue to treat and/or postpone diseases associated with ROS or ER stress. In the findings of Feng et al., ${ }^{52}$ they indicated that curcumin could inhibit the PERK-eIF $2 \alpha-$ CHOP axis of the ER stress response through the activation of SIRT1 in tertbutyl hydroperoxide (TBHP)-treated rat chondrocytes and ameliorated osteoarthritis development in vivo. Together, the previous studies focused either on the anti-inflammatory effect of curcumin in vitro under various stimuli or on its protection against ER stress in other inflammatory diseases. Instead, our study provided the evidence that curcumin alleviated $\mathrm{AD}$ development by reducing inflammatory cytokines and ER stress in brain tissues from ApoE4-Tg mice.

Taken together, we speculated that ApoE4 induced ER stress in mice, activated the inflammatory signaling pathway, led to neuroinflammation in the central nervous system, and affected the learning and memory ability of animals. In 


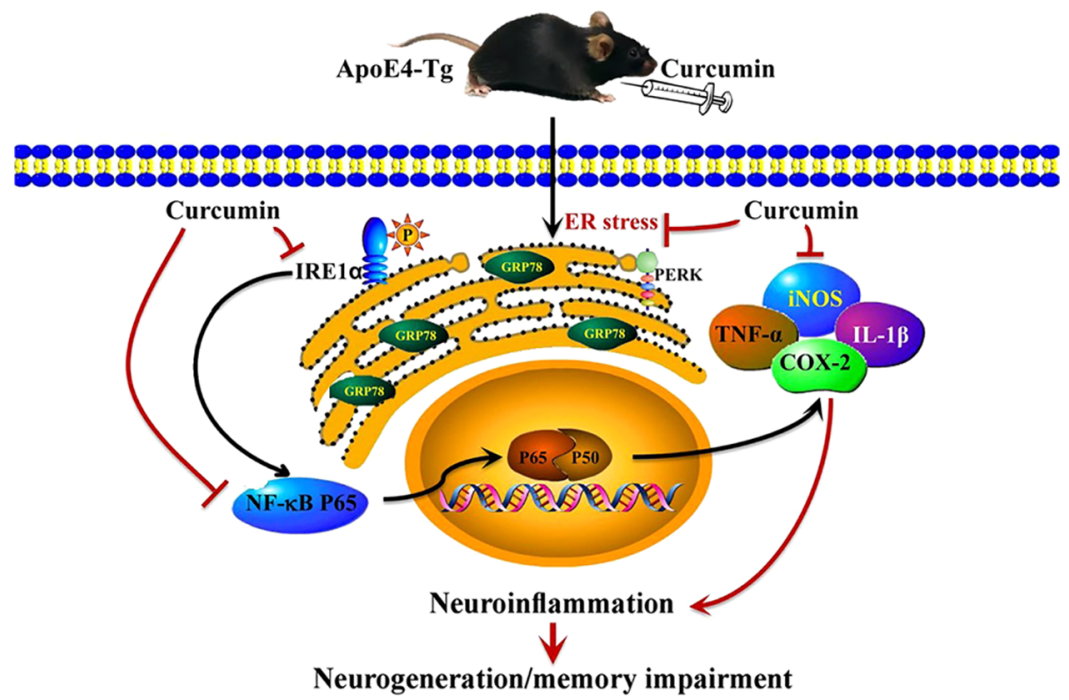

Figure 7. Potential mechanisms of curcumin involved in improving cognitive dysfunction in ApoE4-Tg mice. ApoE4 may trigger ER stress and then activate the inflammatory signaling pathway to trigger neuroinflammation in the nervous system, leading to the occurrence of $\mathrm{AD}$. However, curcumin effectively inhibited the neuroinflammation caused by ApoE4 by inhibiting the occurrence of ER stress and inhibiting the activation of the inflammatory signaling pathway and finally protected the learning and memory ability of ApoE4-Tg mice.

contrast, curcumin treatment inhibited the occurrence of ER stress and activation of the NF- $\kappa \mathrm{B}$ signaling pathway in ApoE4Tg mice, indicative of improved neuroinflammation and finally leading to better learning and memory ability in ApoE4-Tg mice (Figure 7).

\section{MATERIALS AND METHODS}

4.1. Compounds and Reagents. Curcumin (purity $>98.0 \%$ ) was obtained from Sigma Technology (Danvers). Nissl staining solution was purchased from Beyotime Biotechnology Co., Ltd. (Shanghai, China). TNF- $\alpha$ and IL$1 \beta$ enzyme-linked immunosorbent assay (ELISA) kits were provided by Elabscience Biotechnology Co., Ltd. (Wuhan, China). 4',6-Diamidino-2-phenylindole (DAPI) was purchased from Solarbio Science (Beijing, China). The ApoE4, COX-2, iNOS, NF- $\kappa$ Bp65, p-NF- $\kappa$ Bp65, GRP78, IRE $1 \alpha, \beta$-actin, and IgG primary antibodies and Alexa Fluor 488 fluorescent secondary antibodies were all purchased from Abcam and were used at the indicated dilutions for western blotting and immunostaining.

4.2. Animals. ApoE4 transgenic mice (SCXK2016-0004) with a C57BL/6 J background and C57BL/6 J mice were obtained from Jiangsu ALF Biotechnology Co., Ltd. (Nanjing, China). The C57BL/6 J mice were used as wild-type control mice. ApoE4 mice were genotyped by PCR amplification of genomic DNA extracted from tail snips.

Animals were housed in a facility under a $12 \mathrm{~h} / 12 \mathrm{~h}$ light/ dark cycle and had access to food and water ad libitum. Male and female transgenic (Tg) or wild-type (WT) mice aged 8 months were used in all studies. All animal experiments utilized protocols that were performed according to the Guidelines for the Care and Use of Laboratory Animals (Ministry of Science and Technology of the People's Republic of China) and the Ethics Committee of Henan University.

4.3. Experimental Design. The mice were divided into the following four groups, and there were eight mice in each group, half male and half female: WT-control, ApoE4-Tg, ApoE4 + curcumin $(40 \mathrm{mg} / \mathrm{kg})$, and WT + curcumin (40 mg/ $\mathrm{kg}$ ). Curcumin was dissolved in $<1 \%$ dimethyl sulfoxide
(DMSO) and injected intraperitoneally once a day for 3 weeks. After 3 weeks of continuous administration, the Morris water maze test was carried out for 4 consecutive days. The probe test was performed $24 \mathrm{~h}$ (i.e. day 5) after the navigation test (Figure 8). The weight of mice was $25-30 \mathrm{~g}$, and the effect of curcumin on body weight is shown in the Supporting Information (Figure S2).

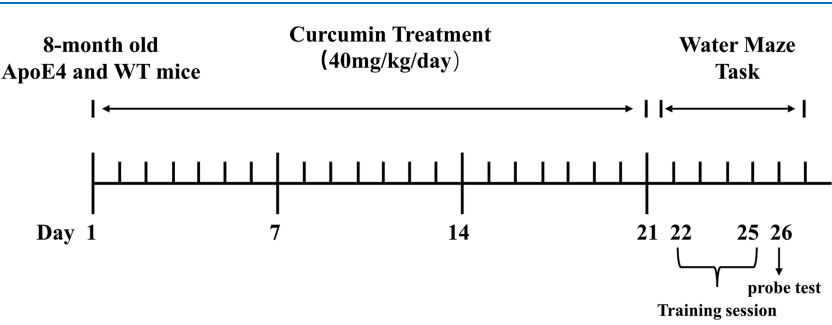

Figure 8. Overview of the protocol of the experimental procedures.

4.4. Morris Water Maze Test. The Morris water maze test was used to assess spatial learning and memory in this study. The mice were placed in a circular pool $(40 \mathrm{~cm}$ high, $120 \mathrm{~cm}$ diameter) for a series of tests at a temperature of $22-25{ }^{\circ} \mathrm{C}$. The mice were trained to find an escape platform (diameter 8 $\mathrm{cm}$ ) that was submerged $1-1.5 \mathrm{~cm}$ below the surface of the water. Each trial lasted $90 \mathrm{~s}$ or ended immediately after the mice reached the underwater platform. If the animal failed to find the platform within $90 \mathrm{~s}$, it was guided to the platform and allowed to stay there for 15-20 s. Each animal had three random starting points, and the test was repeated three times a day for four days (days 1-4). The swimming pattern of the mice was recorded by a camera placed above the center of the pool, and the escape latency, escape distance, and swimming speed of the mice were assessed by a ZS-001 video analysis system (Beijing, China).

The probe test was performed $24 \mathrm{~h}$ (i.e., day 5) after the navigation test for assessing memory consolidation. During the experiment, the platform was removed from the pool, and the mice were allowed to swim freely. The swimming pattern of each mouse was monitored and recorded using a ZS-001 
system (Beijing, China) for $90 \mathrm{~s}$. The time spent in the target quadrant and the number of passes through the platform were used to measure spatial memory. ${ }^{53}$

4.5. Collection and Preservation of Brain Tissues. After the probe test, the mice were anesthetized and then infused with phosphate-buffered saline (PBS). The brain was immediately removed from the skull and divided into two parts: one part was used for immunostaining and the other was used for biochemical analysis. The brain tissues for biochemical analysis were dissected on ice, and all brain tissues were preserved at $-80{ }^{\circ} \mathrm{C}$ for biochemical analysis.

4.6. Nissl Staining. The brain tissues were fixed in $4 \%$ paraformaldehyde for $24 \mathrm{~h}$ and then dehydrated with an alcohol gradient. Then, coronal sections $(25 \mu \mathrm{m})$ were obtained with a cryostat slicer and stained with cresyl violet solution $(0.1 \%)$ for $8 \mathrm{~min}$. Next, the slices were dehydrated with a series of ethanol solutions with increasing concentration (50, 70, 90, and $100 \%$ ethanol) and soaked in a mixture of complete alcohol and xylene (1:1) for $10 \mathrm{~min}$. The sections were cleaned in xylene for $5 \mathrm{~min}$, observed under a microscope, and photographed.

4.7. Immunofluorescence Staining. Sections were sealed with $3 \% \mathrm{H}_{2} \mathrm{O}_{2}$ and $0.3 \%$ Triton-X 100 in PBS at room temperature for $1 \mathrm{~h}$, and then antigen repair was performed with citric acid buffer. Following overnight incubation with primary antibodies at $4{ }^{\circ} \mathrm{C}$ and an Alexa Fluor 488-conjugated goat anti-rabbit IgG secondary antibody at room temperature for $2 \mathrm{~h}$, the cell nuclei were stained with DAPI for $10 \mathrm{~min}$. The samples were then examined using a fluorescence microscope (Nikon, Tokyo, Japan), and the area of immune response was quantified by ImageJ2x (National Institutes of Health, Bethesda, MD).

4.8. Immunohistochemistry. The $5 \mu \mathrm{m}$ slices were sealed with $5 \%$ bovine serum albumin (BSA) and $0.3 \%$ Triton-X 100 in PBS for $1 \mathrm{~h}$ at room temperature, and then antigen repair was performed with citric acid buffer. Slices were incubated with the primary antibodies overnight at $4{ }^{\circ} \mathrm{C}$, and goat antirabbit IgG secondary antibodies were added for $30 \mathrm{~min}$. Then, diaminobenzidine was used for imaging, and hematoxylin was used for staining. The immunopositive cells were stained brown. ${ }^{54}$ The positive expression of the specific protein was photographed and recorded under an inverted microscope, and the positive area rate was calculated by Image $2 x$.

4.9. ELISA Assay. The levels of TNF- $\alpha$ and IL- $1 \beta$ in the brain tissues were measured using ELISA kits according to the manufacturer's instructions. Flash-frozen brains from treated mice were homogenized and lysed to perform ELISA using the tissue lysate. Absorbance values were determined by a microplate reader, and sample concentration was analyzed using the standard curves.

4.10. Western Blotting. Protein concentrations were measured using a protein assay kit according to the manufacturer's instructions. The extracted proteins were separated by SDS-polyacrylamide gel electrophoresis and then transferred to a membrane for $30 \mathrm{~min}$. The membrane was blocked with $5 \%$ skim milk and then incubated at $4{ }^{\circ} \mathrm{C}$ overnight with the following primary antibodies: GRP78, IRE $1 \alpha$, p65, p-p65, $\beta$-actin, or lamin B. Then, the membrane was washed three times with TBST and incubated with the secondary antibody for $2 \mathrm{~h}$. The ECL method was used to develop the film, and dark room exposure analysis and Image $2 \mathrm{x}$ analysis software were used to analyze the grayscale values. 55
4.11. Statistical Analysis. Data are presented as the mean \pm SEM. Comparisons between groups were performed with one-way ANOVA followed by Dunnett's post hoc test. $p<0.05$ was considered statistically significant.

\section{ASSOCIATED CONTENT}

Supporting Information

The Supporting Information is available free of charge at https://pubs.acs.org/doi/10.1021/acsomega.0c04810.

Chemical structure of curcumin; the effect of curcumin on body weight in mice; the other two repeats of western blotting and IHC; plaque burden developed in ApoE4-Tg mice; the representative image and quantification analysis of P-PERK expression; and the other two repeats of western expression of P-PERK (PDF)

\section{AUTHOR INFORMATION}

\section{Corresponding Authors}

Xinmei Xie - School of Pharmacy, Henan University, Kaifeng 475004, Henan, China; Email: xxm@vip.henu.edu.cn

Xiaobin Pang - School of Pharmacy, Henan University, Kaifeng 475004, Henan, China; Phone: +86475 2388 0680; Email: pxb@vip.henu.edu.cn

\section{Authors}

Jiejian Kou - School of Pharmacy, Henan University, Kaifeng 475004, Henan, China; 10 orcid.org/0000-0001-91374769

Minghui Wang - School of Pharmacy, Henan University, Kaifeng 475004, Henan, China

Junzhuo Shi - School of Pharmacy, Henan University, Kaifeng 475004, Henan, China

Haiyu Zhang - School of Pharmacy, Henan University, Kaifeng 475004, Henan, China

Xiaohui Pu - School of Pharmacy, Henan University, Kaifeng 475004, Henan, China

Shiyong Song - School of Pharmacy, Henan University, Kaifeng 475004, Henan, China; orcid.org/0000-00032208-608X

Cuiling Yang - State Key Laboratory of Crop Stress Adaptation and Improvement, Henan University, Kaifeng 475004, Henan, China

Yi Yan - Institute for Cardiovascular Prevention (IPEK), Ludwig-Maximilians-University Munich, Munich 80331, Germany; DZHK (German Centre for Cardiovascular Research), Partner Site Munich Heart Alliance, Munich 80336, Germany; 이이.org/0000-0003-2860-3252

Yvonne Döring - Institute for Cardiovascular Prevention (IPEK), Ludwig-Maximilians-University Munich, Munich 80331, Germany; DZHK (German Centre for Cardiovascular Research), Partner Site Munich Heart Alliance, Munich 80336, Germany; Department of Angiology, Swiss Cardiovascular Center, Inselspital, Bern University Hospital, University of Bern, Bern 3010, Switzerland

Complete contact information is available at:

https://pubs.acs.org/10.1021/acsomega.0c04810

\section{Author Contributions}

\#.K. and M.W. contributed equally to this work.

\section{Funding}

This study was supported by grants from the National Natural Science Foundation of China (31872311) and the Science 
Foundation for Outstanding Young Scholars of Henan Province (No. 212300410027).

\section{Notes}

The authors declare no competing financial interest.

\section{ABBREVIATIONS USED}

$\mathrm{A} \beta \quad \beta$-amyloid peptides

$\mathrm{AD} \quad$ Alzheimer's disease

ApoE4 apolipoprotein E4

ApoE4-Tg ApoE4 transgenic

APs amyloid plaques

ANOVA analysis of variance

BSA bovine serum albumin

Cur curcumin

DAPI 4',6-diamidino-2-phenylindole

DMSO dimethyl sulfoxide

DNA deoxyribonucleic acid

ER endoplasmic reticulum

ELISA enzyme-linked immunosorbent assay

NFTs neurofibrillary tangles

GRP78 glucose-regulated protein 78

IgG immunoglobulin $\mathrm{G}$

IL-1 $\beta \quad$ interleukin- $1 \beta$

iNOS inducible nitric oxide synthase

IRE1 $\alpha$ inositol-requiring enzyme 1

PBS phosphate-buffered saline

SDS sodium dodecyl sulfate

TNF- $\alpha$ tumor necrosis factor- $\alpha$

WT wild type

\section{REFERENCES}

(1) Lane, C. A.; Hardy, J.; Schott, J. M. Alzheimer's disease. Eur. J. Neurol. 2018, 25, 59-70.

(2) Alzheimer's Association. Alzheimer's disease facts and figures. Alzheimer's Dementia 2016, 12, 459-509.

(3) Pallo, S. P.; DiMaio, J.; Cook, A.; Nilsson, B.; Johnson, G. Mechanisms of tau and $\mathrm{A} \beta$-induced excitotoxicity. Brain Res. 2016, 1634, 119-131.

(4) Zhao, F.; Zhang, J.; Chang, N. Epigenetic modification of Nrf2 by sulforaphane increases the antioxidative and anti-inflammatory capacity in a cellular model of Alzheimer's disease. Eur. J. Pharmacol. 2018, 824, 1-10.

(5) Zhu, S.; Wang, J.; Zhang, Y.; He, J.; Kong, J.; Wang, J. F.; Li, X. $M$. The role of neuroinflammation and amyloid in cognitive impairment in an APP/PS1 transgenic mouse model of Alzheimer's disease. CNS Neurosci. Ther. 2017, 23, 310-320.

(6) Volicer, L. Physiological and pathological functions of betaamyloid in the brain and alzheimer's disease: A review. Chin. J. Physiol. 2020, 63, 95-100.

(7) Liang, T.; Xue, F.; Hang, W.; Wen, B.; Zhang, Q.; Chen, J.; Liu, X.; Chen, J. Neuron-Specific Apolipoprotein E4 (1-272) Fragment Induces Tau Hyperphosphorylation and Axonopathy via Triggering Endoplasmic Reticulum Stress. J. Alzheimer's Dis. 2019, 71, 597-611.

(8) Lin, Y. T.; Seo, J.; Gao, F.; Feldman, H. M.; Wen, H. L.; Penney, J.; Cam, H. P.; Gjoneska, E.; Raja, W. K.; Cheng, J.; Rueda, R.; Kritskiy, O.; Abdurrob, F.; Peng, Z.; Milo, B.; Yu, C. J.; Elmsaouri, S.; Dey, D.; Ko, T.; Yankner, B. A.; Tsai, L. H. APOE4 Causes Widespread Molecular and Cellular Alterations Associated with Alzheimer's Disease Phenotypes in Human iPSC-Derived Brain Cell Types. Neuron 2018, 98, 1141-1154.

(9) Esquerda-Canals, G.; Montoliu-Gaya, L.; Güell-Bosch, J.; Villegas, S. Mouse Models of Alzheimer's Disease. J. Alzheimer's Dis. 2017, 57, 1171-1183.
(10) Theendakara, V.; Peters-Libeu, C. A.; Bredesen, D. E.; Rao, R. V. Transcriptional Effects of ApoE4: Relevance to Alzheimer's Disease. Mol. Neurobiol. 2018, 55, 5243-5254.

(11) Uddin, M. S.; Kabir, M. T.; Al Mamun, A.; Abdel-Daim, M. M.; Barreto, G. E.; Ashraf, G. M. APOE and Alzheimer's Disease: Evidence Mounts that Targeting APOE4 may Combat Alzheimer's Pathogenesis. Mol. Neurobiol. 2019, 56, 2450-2465.

(12) Huang, S.; Xing, Y.; Liu, Y. Emerging roles for the ER stress sensor IRE $1 \alpha$ in metabolic regulation and disease. J. Biol. Chem. 2019, 294, 18726-18741.

(13) Olivera-Perez, H. M.; Lam, L.; Dang, J.; Jiang, W.; Rodriguez, F.; Rigali, E.; Weitzman, S.; Porter, V.; Rubbi, L.; Morselli, M.; Pellegrini, M.; Fiala, M. Omega-3 fatty acids increase the unfolded protein response and improve amyloid- $\beta$ phagocytosis by macrophages of patients with mild cognitive impairment. FASEB J. 2017, 31, 4359-4369.

(14) Gerakis, Y.; Hetz, C. Emerging roles of ER stress in the etiology and pathogenesis of Alzheimer's disease. FEBS J. 2018, 285, 9951011.

(15) Nelson, K. M.; Dahlin, J. L.; Bisson, J.; Graham, J.; Pauli, G. F.; Walters, M. A. The Essential Medicinal Chemistry of Curcumin. J. Med. Chem. 2017, 60, 1620-1637.

(16) Potter, P. E. Curcumin: a natural substance with potential efficacy in Alzheimer's disease. J. Exp. Pharmacol. 2013, 5, 23-31.

(17) Shehzad, A.; Qureshi, M.; Anwar, M. N.; Lee, Y. S. Multifunctional Curcumin Mediate Multitherapeutic Effects. J. Food Sci. 2017, 82, 2006-2015.

(18) Goel, A.; Kunnumakkara, A. B.; Aggarwal, B. B. Curcumin as "Curecumin": from kitchen to clinic. Biochem. Pharmacol. 2008, 75, 787-809.

(19) Reddy, P. H.; Manczak, M.; Yin, X.; Grady, M. C.; Mitchell, A.; Tonk, S.; Kuruva, C. S.; Bhatti, J. S.; Kandimalla, R.; Vijayan, M.; Kumar, S.; Wang, R.; Pradeepkiran, J. A.; Ogunmokun, G.; Thamarai, K.; Quesada, K.; Boles, A.; Reddy, A. P. Protective Effects of Indian Spice Curcumin Against Amyloid- $\beta$ in Alzheimer's Disease. J. Alzheimer's Dis. 2018, 61, 843-866.

(20) Tang, M.; Taghibiglou, C. The Mechanisms of Action of Curcumin in Alzheimer's Disease. J. Alzheimer's Dis. 2017, 58, 10031016.

(21) Ho, Y. S.; Yang, X.; Lau, J. C.; Hung, C. H.; Wuwongse, S.; Zhang, Q.; Wang, J.; Baum, L.; So, K. F.; Chang, R. C. Endoplasmic reticulum stress induces tau pathology and forms a vicious cycle: implication in Alzheimer's disease pathogenesis. J. Alzheimer's Dis. 2012, 28, 839-854.

(22) Yamazaki, Y.; Zhao, N.; Caulfield, T. R.; Liu, C. C.; Bu, G.; Apolipoprotein, E. and Alzheimer disease: pathobiology and targeting strategies. Nat. Rev. Neurol. 2019, 15, 501-518.

(23) Sun, G. Z.; He, Y. C.; Ma, X. K.; Li, S. T.; Chen, D. J.; Gao, M.; Qiu, S. F.; Yin, J. X.; Shi, J.; Wu, J. Hippocampal synaptic and neural network deficits in young mice carrying the human APOE4 gene. CNS Neurosci. Ther. 2017, 23, 748-758.

(24) Tachibana, M.; Holm, M. L.; Liu, C. C.; Shinohara, M.; Aikawa, T.; Oue, H.; Yamazaki, Y.; Martens, Y. A.; Murray, M. E.; Sullivan, P. M.; Weyer, K.; Glerup, S.; Dickson, D. W.; Bu, G.; Kanekiyo, T. APOE4-mediated amyloid- $\beta$ pathology depends on its neuronal receptor LRP1. J. Clin. Invest. 2019, 129, 1272-1277.

(25) Lewandowski, C. T.; Maldonado Weng, J.; LaDu, M. J. Alzheimer's disease pathology in APOE transgenic mouse models: The Who, What, When, Where, Why, and How. Neurobiol. Dis. 2020, 139, No. 104811.

(26) Shi, Y.; Yamada, K.; Liddelow, S. A.; Smith, S. T.; Zhao, L.; Luo, W.; Tsai, R. M.; Spina, S.; Grinberg, L. T.; Rojas, J. C.; Gallardo, G.; Wang, K.; Roh, J.; Robinson, G.; Finn, M. B.; Jiang, H.; Sullivan, P. M.; Baufeld, C.; Wood, M. W.; Sutphen, C.; Holtzman, D. M. ApoE4 markedly exacerbates tau-mediated neurodegeneration in a mouse model of tauopathy. Nature 2017, 549, 523-527.

(27) Rohn, T. T. Is apolipoprotein E4 an important risk factor for vascular dementia? Int. J. Clin. Exp. Pathol. 2014, 7, 3504-3511. 
(28) Johnson, L. A.; Kim, H. S.; Knudson, M. J.; Nipp, C. T.; Yi, X.; Maeda, N. Diabetic atherosclerosis in $\mathrm{APOE}^{*} 4$ mice: synergy between lipoprotein metabolism and vascular inflammation. J. Lipid Res. 2013, 54, 386-396.

(29) Buchanan, H.; Mackay, M.; Palmer, K.; Tothová, K.; Katsur, M.; Platt, B.; Koss, D. J. Synaptic Loss, ER Stress and NeuroInflammation Emerge Late in the Lateral Temporal Cortex and Associate with Progressive Tau Pathology in Alzheimer's Disease. Mol. Neurobiol. 2020, 57, 3258-3272.

(30) Li, K.; Wei, Q.; Liu, F. F.; Hu, F.; Xie, A. J.; Zhu, L. Q.; Liu, D. Synaptic Dysfunction in Alzheimer's Disease: $\mathrm{A} \beta$, Tau, and Epigenetic Alterations. Mol. Neurobiol. 2018, 55, 3021-3032.

(31) Calsolaro, V.; Edison, P. Neuroinflammation in Alzheimer's disease: Current evidence and future directions. Alzheimer's Dementia 2016, 12, 719-732.

(32) Hoozemans, J. J.; Stieler, J.; van Haastert, E. S.; Veerhuis, R.; Rozemuller, A. J.; Baas, F.; Eikelenboom, P.; Arendt, T.; Scheper, W. The unfolded protein response affects neuronal cell cycle protein expression: implications for Alzheimer's disease pathogenesis. Exp. Gerontol. 2006, 41, 380-386.

(33) Salminen, A.; Kaarniranta, K.; Kauppinen, A. ER stress activates immunosuppressive network: implications for aging and Alzheimer's disease. J. Mol. Med. 2020, 98, 633-650.

(34) Cash, J. G.; Kuhel, D. G.; Basford, J. E.; Jaeschke, A.; Chatterjee, T. K.; Weintraub, N. L.; Hui, D. Y. Apolipoprotein E4 impairs macrophage efferocytosis and potentiates apoptosis by accelerating endoplasmic reticulum stress. J. Biol. Chem. 2012, 287, 27876-27884.

(35) Duran-Aniotz, C.; Cornejo, V. H.; Espinoza, S.; Ardiles, Á. O.; Medinas, D. B.; Salazar, C.; Fol, A.; Gajardo, I.; Thielen, P.; Iwawaki, T.; Scheper, W.; Soto, C.; Palacios, A. G.; Hoozemans, J.; Hetz, C. IRE1 signaling exacerbates Alzheimer's disease pathogenesis. Acta Neuropathol. 2017, 134, 489-506.

(36) Cui, W.; Wang, S.; Wang, Z.; Wang, Z.; Sun, C.; Zhang, Y. Inhibition of PTEN Attenuates Endoplasmic Reticulum Stress and Apoptosis via Activation of PI3K/AKT Pathway in Alzheimer's Disease. Neurochem. Res. 2017, 42, 3052-3060.

(37) Lanzillotta, C.; Tramutola, A.; Meier, S.; Schmitt, F.; Barone, E.; Perluigi, M.; Di Domenico, F.; Abisambra, J. F. Early and Selective Activation and Subsequent Alterations to the Unfolded Protein Response in Down Syndrome Mouse Models. J. Alzheimer's Dis. 2018, 62, 347-359.

(38) Song, S.; Tan, J.; Miao, Y.; Zhang, Q. Crosstalk of ER stressmediated autophagy and ER-phagy: Involvement of UPR and the core autophagy machinery. J. Cell. Physiol. 2018, 233, 3867-3874.

(39) Gong, J.; Wang, X. Z.; Wang, T.; Chen, J. J.; Xie, X. Y.; Hu, H.; Yu, F.; Liu, H. L.; Jiang, X. Y.; Fan, H. D. Molecular signal networks and regulating mechanisms of the unfolded protein response. $J$. Zhejiang Univ., Sci., B 2017, 18, 1-14.

(40) Chen, J.; Zhang, M.; Zhu, M.; Gu, J.; Song, J.; Cui, L.; Liu, D.; Ning, Q.; Jia, X.; Feng, L. Paeoniflorin prevents endoplasmic reticulum stress-associated inflammation in lipopolysaccharide-stimulated human umbilical vein endothelial cells via the IRE $1 \alpha / \mathrm{NF}-\kappa \mathrm{B}$ signaling pathway. Food Funct. 2018, 9, 2386-2397.

(41) Zha, X.; Yue, Y.; Dong, N.; Xiong, S. Endoplasmic Reticulum Stress Aggravates Viral Myocarditis by Raising Inflammation Through the IRE1-Associated NF- $\kappa$ B Pathway. Can. J. Cardiol. 2015, 31, $1032-1040$.

(42) Li, Q.; Tian, Z.; Wang, M.; Kou, J.; Wang, C.; Rong, X.; Li, J.; $\mathrm{Xie}, \mathrm{X}$.; Pang, X. Luteoloside attenuates neuroinflammation in focal cerebral ischemia in rats via regulation of the PPAR $\gamma / \mathrm{Nrf} 2 / \mathrm{NF}-\kappa \mathrm{B}$ signaling pathway. Int. Immunopharmacol. 2019, 66, 309-316.

(43) Wang, L.; Wang, M.; Hu, J.; Shen, W.; Hu, J.; Yao, Y.; Wang, X.; Afzal, C. M.; Ma, R.; Li, G. Protective effect of 3H-1, 2-dithiole-3thione on cellular model of Alzheimer's disease involves Nrf2/ARE signaling pathway. Eur. J. Pharmacol. 2017, 795, 115-123.

(44) Conejero-Goldberg, C.; Hyde, T. M.; Chen, S.; Herman, M. M.; Kleinman, J. E.; Davies, P.; Goldberg, T. E. Cortical Transcriptional Profiles in APOE4 Carriers with Alzheimer's Disease: Patterns of Protection and Degeneration. J. Alzheimer's Dis. 2015, 48, 969978

(45) Zhu, X.; Shi, J.; Li, H. Liquiritigenin attenuates high glucoseinduced mesangial matrix accumulation, oxidative stress, and inflammation by suppression of the NF- $\kappa \mathrm{B}$ and NLRP3 inflammasome pathways. Biomed. Pharmacother. 2018, 106, 976-982.

(46) Shabab, T.; Khanabdali, R.; Moghadamtousi, S. Z.; Kadir, H. A.; Mohan, G. Neuroinflammation pathways: a general review. Int. J. Neurosci. 2017, 127, 624-633.

(47) Goel, A.; Boland, C. R.; Chauhan, D. P. Specific inhibition of cyclooxygenase-2 (COX-2) expression by dietary curcumin in HT-29 human colon cancer cells. Cancer Lett. 2001, 172, 111-118.

(48) Zhang, J.; Zheng, Y.; Luo, Y.; Du, Y.; Zhang, X.; Fu, J. Curcumin inhibits LPS-induced neuroinflammation by promoting microglial M2 polarization via TREM2/ TLR4/ NF- $\kappa$ B pathways in BV2 cells. Mol. Immunol. 2019, 116, 29-37.

(49) Wang, M.; Kou, J.; Wang, C.; Yu, X.; Xie, X.; Pang, X. Curcumin inhibits APOE4-induced injury by activating peroxisome proliferator-activated receptor- $\gamma(\mathrm{PPAR} \gamma)$ in SH-SY5Y cells. Iran. J. Basic Med. Sci. 2020, 1576-1583.

(50) Zheng, K. M.; Zhang, J.; Zhang, C. L.; Zhang, Y. W.; Chen, X. C. Curcumin inhibits appoptosin-induced apoptosis via upregulating heme oxygenase-1 expression in SH-SY5Y cells. Acta Pharmacol. Sin. 2015, 36, 544-552.

(51) Chhunchha, B.; Fatma, N.; Kubo, E.; Rai, P.; Singh, S. P.; Singh, D. P. Curcumin abates hypoxia-induced oxidative stress basedER stress-mediated cell death in mouse hippocampal cells (HT22) by controlling Prdx6 and NF- $\kappa$ B regulation. Am. J. Physiol.: Cell Physiol. 2013, 304, C636-C655.

(52) Feng, K.; Ge, Y.; Chen, Z.; Li, X.; Liu, Z.; Li, X.; Li, H.; Tang, T.; Yang, F.; Wang, X. Curcumin Inhibits the PERK-eIF $2 \alpha-C H O P$ Pathway through Promoting SIRT1 Expression in Oxidative Stressinduced Rat Chondrocytes and Ameliorates Osteoarthritis Progression in a Rat Model. Oxid. Med. Cell. Longevity 2019, No. 8574386 .

(53) Rajashri, K.; Mudhol, S.; Serva Peddha, M.; Borse, B. B. Neuroprotective Effect of Spice Oleoresins on Memory and Cognitive Impairment Associated with Scopolamine-Induced Alzheimer's Disease in Rats. ACS Omega 2020, 5, 30898-30905.

(54) Yan, W.; Deng, W.; Cui, S. DNA methylation and cerebellar development, the regulation of Notch and Shh pathway. Ital. J. Zool. 2016, 83, 34-42.

(55) Cheng, X. S.; Li, M. S.; Du, J.; Jiang, Q. Y.; Wang, L.; Yan, S. Y.; Yu, D. M.; Deng, J. B. Neuronal apoptosis in the developing cerebellum. Anat., Histol., Embryol. 2011, 40, 21-27. 